\title{
Análisis de la influencia de la privatización de aeropuertos en el pronóstico de la demanda de pasajeros. El caso de Colombia
}

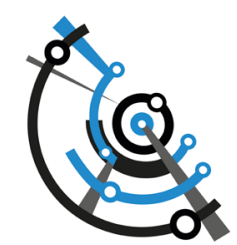

\author{
- Oscar Díaz Olariaga \\ Facultad de Ingeniería Civil, Universidad Santo Tomás, Colombia.
}

\author{
Edwin Girón Amaya \\ Facultad de Estadística, Universidad Santo Tomás, Colombia.
}

Recibido: 12 de enero de 2018. Aceptado: 30 de julio de 2019.

\begin{abstract}
Resumen
En este trabajo se analiza el impacto de la privatización de aeropuertos en el desarrollo futuro de la demanda de pasajeros en dichos aeropuertos privatizados, y como marco de aplicación se utiliza la red aeroportuaria colombiana, la cual experimenta, desde la liberación de la industria del transporte aéreo (1991), un importante y extenso desarrollo de concesiones. La investigación se centra sobre la influencia de la privatización del aeropuerto en el pronóstico a corto plazo de la demanda de pasajeros. Para estimar los efectos que ejerce sobre la demanda de pasajeros la privatización de los aeropuertos se desarrolla un modelo de series de tiempo empleando la metodología de Box-Jenkins usando modelos ARIMAX. Los principales resultados muestran pronósticos de crecimiento positivo de la demanda en la mayoría de los aeropuertos analizados.
\end{abstract}

Palabras clave: Transporte Aéreo. Demanda. Privatización de Aeropuerto. Pronóstico. Colombia.

\section{Analysis of the influence of the privatization of airports on the forecast of passenger demand. The case of Colombia}

\footnotetext{
Abstract

This paper analyzes the impact of airport privatization on the future development of passenger demand in these privatized airports. The Colombian airport network is used as an application framework because of its important and extensive development of concessions from the liberation of the transport industry in 1991. The research focuses on the influence of airport privatization on the short-term forecast of passenger demand. To estimate the effects on passenger demand of airport privatization, a time
} 
series model is developed using the Box-Jenkins methodology, i.e. ARIMAX models. The main results show forecasts of positive growth in demand at most of the airports analyzed.

Keywords: Air Transport. Demand. Airport Privatization. Forecast. Colombia.

Palavras-chave: Transporte Aéreo. Demanda. Privatização de aeroporto. Previsão. Colômbia.

\section{Introducción}

El transporte aéreo colombiano está experimentado un dinámico desarrollo que comenzó hace dos décadas y media. Este periodo coincide con el inicio de una continuada implementación de políticas públicas elaboradas específicamente para el sector del transporte aéreo. El crecimiento del tráfico aéreo en Colombia ha venido reforzado desde mediados de la década de 1990 por una política pública de liberalización de la industria aeroportuaria, dando entrada al capital privado en la modernización, ampliación y actualización de las infraestructuras del transporte aéreo, concesionando, en varias fases denominadas generaciones, los aeropuertos de mayor tráfico del país (Díaz Olariaga, 2016a).

En esta línea, algunos estudios sugieren que los aeropuertos privatizados, es decir operado por una empresa privada, logran una mayor eficiencia operativa que los gestionados por el sector público (Oum et al., 2008 y 2006). Y por otro lado, algunos estudios (Rolim et al., 2016) analizan la cuestión de la incidencia de la privatización del aeropuerto en la demanda de pasajeros.

En Colombia, el proceso de privatización de un aeropuerto es uno relativamente largo que tiene asociado unas etapas o un calendario. Ahora bien, en el presente estudio el hito que marca el cambio de la gestión u operación del aeropuerto de pública a privada (nunca de la propiedad), es cuando el Estado o sector público concede (vía proceso licitatorio) al operador privado la concesión del aeropuerto. El siguiente paso es la entrega (formal) del aeropuerto, que puede ser varios meses luego de la concesión (previamente se realiza la firma del contrato de concesión), y a partir de este momento es cuando el operador empieza a modernizar / ampliar el aeropuerto, proceso que puede durar de 1 a 5 años, dependiendo del tamaño del aeropuerto concesionado y de las obras comprometidas. Para el caso de Colombia no se conocen estudios que analicen el comportamiento de la demanda de pasajeros aéreos en periodos de post-privatización de aeropuertos concesionados a nivel de pronósticos. Sólo se encuentra un trabajo (Díaz Olariaga, 2016b) que analiza el desarrollo histórico del tráfico de pasajeros y carga (pre y post liberalización de la industria), el efecto que las políticas públicas sobre dicho desarrollo, el análisis de los indicadores del sector del transporte aéreo, y el impacto de este sector tanto en la economía como en otras industrias relacionadas.

Entonces, para estimar los efectos que ejerce sobre la demanda de pasajeros la privatización de los aeropuertos se desarrolla un modelo de series de tiempo empleando la metodología de Box - Jenkins usando modelos ARIMAX con los cuales se aprovecha la ventaja de tener información exógena que está correlacionada con el comportamiento de las series (Abed et al., 2001). Y finalmente mencionar que el pronóstico de tráfico se realiza a corto plazo (6 años), no sólo por ser lo habitual en la prognosis de tráfico aéreo, sino porque además la relativamente corta serie histórica disponible (24 años) no permite, bajo ciertos criterios de fiabilidad, previsiones a medio y largo plazo.

En el presente trabajo se organiza de la siguiente manera: en primer lugar se realiza la oportuna revisión bibliográfica, para conocer el "estado del arte", e indagar sobre la existencia de investigaciones similares o iguales, y en particular en la región 
latinoamericana y en Colombia. Paso seguido se realiza un análisis del entorno local, es decir, sobre la situación del sistema aeroportuario (y del transporte aéreo) colombiano, partiendo desde la liberalización de la industria, lo que condujo a la privatización, escalonada, de un importante grupo de aeropuertos. En la subsiguiente sección se presenta el desarrollo del marco teórico o conceptual, y del modelo econométrico a utilizar. En la penúltima sección se presentan los resultados y su análisis. Y finalmente en la última sección se mencionan las conclusiones más importantes encontradas en la presente investigación.

\section{Revisión de la bibliografía}

En la literatura relacionada se encuentran muchos estudios relacionados a la política pública, eficiencia operativa y gestión de la capacidad de aeropuertos privatizados (y su comparativa con aeropuertos gestionados de forma pública) (Bettini y Oliveira, 2016; Noruzoliaee et al., 2015; Lin, 2013; Gong et al., 2012; Scotti et al., 2012; Freestone y Baker, 2010; Oum et al., 2008, 2006; Zhang y Zhang, 2003; Advani y Borins, 2001; Parker, 1999). En lo que refiere al análisis de las privatizaciones aeroportuarias en Latinoamérica, existen varios estudios que tratan los mismos tópicos antes citados (Díaz Olariaga, 2016a; Espirito Santo, 2013; Serebrisky, 2012; Mendiola et al., 2011; Carvallo, 2008; Lipovich, 2008; Rico Galeana, 2008; Bosch y García Montalvo, 2003; ICAO, 2003).

Ahora bien, en lo que se refiere a la línea específica de investigación abordada en el presente trabajo, existe un cierto vacío en la literatura científica a nivel regional (Latinoamérica), y muy especialmente a nivel local (Colombia). Sun y Schönfeld (2015) utilizan programas estocásticos para estimar, por un lado, la demanda futura de tráfico, y por otro lado, para optimizar la toma de decisiones en el futuro desarrollo del aeropuerto (inversiones en capacidad), y donde se tienen en cuenta las incertidumbres en las previsiones de tráfico. Todo ello aplicado a mercados o países donde el transporte aéreo está completamente liberalizado. Las variables utilizadas en el modelo son principalmente costes (de capital, de operación, relacionados a los retrasos). Y los pronósticos se realizan para un periodo de 30 años. Singh et al. (2016) realizan un estudio de pronóstico tanto del tráfico aéreo como de la inversión en capacidad aeroportuaria, para un periodo de 20 años, para el conjunto del sistema aeroportuario de la India, y en un contexto de post-liberalización de la industria del transporte aéreo. Ellos utilizan para el cálculo un modelo econométrico, incluyendo variables aeronáuticas (transporte de pasajeros y carga) y económicas, PIB e Índice de Producción Industrial. De sus resultados ellos concluyen que el crecimiento estimado de los pasajeros responderá al crecimiento del PIB, mientras que crecimiento del tráfico de carga responderá al crecimiento de la producción industrial. Scarpel (2013) utiliza modelos expertos para pronosticar la demanda de pasajeros aéreos en el Aeropuerto de Sao Paulo (Brasil) (esta investigación se realizó cuando dicho aeropuerto aún era de gestión pública). El pronóstico se realiza a corto plazo (5 años); el modelo utiliza una variable aeronáutica, pasajeros, y una variable económica, el PIB. Y finalmente, Rolim et al. (2016) analizan el desarrollo de la demanda en aeropuertos recientemente privatizados, aplicado al caso de Brasil; ellos investigan si el proceso de privatización produce un impacto secuencial sobre el tráfico. Y los principales resultados de su estudio sugieren que la privatización produjo un aumento global de la demanda.

En definitiva, el presente trabajo viene a ser el primero en su tipo en analizar la influencia de la privatización del aeropuerto en el pronóstico de la demanda de pasajeros aéreos, y la estimación de la misma, cubriendo de esta manera un vacío en la literatura científica a nivel local (Colombia) y regional (Latinoamérica). 


\section{La liberalización del transporte aéreo en Colombia}

\section{La privatización de aeropuertos en Colombia}

Sobre la gestión de la infraestructura aeroportuaria Colombia ha seguido la tendencia general en América Latina de concesionar la administración / operación (nunca la propiedad) de dichas infraestructuras (Díaz Olariaga, 2016a). Este modelo busca liberar al Estado del alto gasto requerido por la infraestructura aeronáutica. Este proceso está regulado por una ley nacional (Ley 80 de 1993, artículo 30). Por otra parte, el Estado, a través de otra norma (DNP, 1994), consagró el proceso de concesiones de aeropuertos a través de un plan de ordenamiento de la infraestructura aeroportuaria. El documento además de plantear una renovación tecnológica autorizó el proceso de concesión (de la operación) de los aeropuertos a empresas privadas, y consideró la contraprestación económica para el Estado como único elemento para entregar la concesión. A esta contraprestación se le definió como destino: a) alimentar el fondo de compensación aeronáutica para subsidiar a los aeropuertos no rentables, b) financiar nuevas inversiones en dichos aeropuertos y c) financiar los servicios de tráfico aéreo y seguridad. Lo anterior significaba paralelamente que la Autoridad de Aeronáutica Civil de Colombia (en adelante Aerocivil) pudiera concentrar sus labores en la operación, control, desarrollo y mantenimiento del sistema de espacio aéreo y la supervisión de las operaciones aéreas. Mencionar que Aerocivil es el ente público propietario de todos los aeropuertos (concesionados o no) y ostenta las competencias de gestión de todos los aeropuertos no concesionados, del control de tráfico aéreo, de la seguridad aérea, y también es el organismo regulador (tanto de los aeropuertos concesionados como del sector aerocomercial y de toda actividad de aviación civil en el país).

Entonces, desde mediados de la década de 1990, y en cuatro fases temporales, denominadas generaciones, el gobierno colombiano, a través Aerocivil, entregó en concesión varios aeropuertos del país, un total de 18, los más grandes e importantes de los 75 que administra la autoridad pública aeronáutica (Díaz Olariaga, 2016b), con el fin de obtener una mejor administración, modernización y expansión, operación, explotación comercial y mantenimiento de las terminales aéreas de mayor uso. Bajo el objetivo planteado de las concesiones aeroportuarias del país, la empresa, sociedad, consorcio o ente concesionario de los aeropuertos se desempeña únicamente como administrador (u operador) del mismo.

La primera generación de concesiones se realizó bajo un modelo de ingresos mínimos garantizados al concesionario, con lo cual éste no asumía ningún riesgo. En esta generación se ceden al concesionario todos los ingresos regulados y no regulados de los aeropuertos, a cambio de una contraprestación fija para el Estado. También se establecía para el concesionario la responsabilidad del manejo, mantenimiento y operación del terminal, pista, rampa, instalaciones aeroportuarias y ayudas audiovisuales de aproximación y zonas accesorias. En esta generación las concesiones no contenían un plan maestro, lo que hizo imposible que se identificaran y cuantificaran las inversiones necesarias y por lo tanto, las que se realizaron fueron en obras menores de la terminal, determinadas por el mismo concesionario. De esta manera, la vinculación del capital privado a la infraestructura fue insignificante (SIC, 2013).

A partir de la segunda generación de concesiones, concretadas en el período 20002007, las contraprestaciones para el Estado se distribuyen entre unos cargos fijos y unos variables sobre el ingreso bruto del concesionario. Este cambio en los términos de la privatización de los aeropuertos se hizo necesario debido a que los ingresos que estaba recibiendo el Estado de ciertos aeropuertos no eran suficientes para llevar a cabo las inversiones que se tenían previstas. Así mismo, se previeron responsabilidades dependiendo de los diferentes agentes involucrados en el proceso y como consecuencia 
se hizo una reasignación de los riesgos. El concesionario quedó a cargo de una serie de inversiones obligatorias y de rehabilitación vinculadas a condiciones de demanda a través de los planes maestros en el que establecían las necesidades a corto, mediano y largo plazo, con una aproximación en costos de las mismas. Éstas últimas debían llevarse a cabo dentro de los primeros doce meses a partir del inicio del contrato, de lo contrario entrarían a aplicar determinadas sanciones (Díaz Olariaga, 2016a).

En la tercera generación de concesiones, año 2010, se mantienen y mejoran las condiciones de la segunda. No obstante, las diferencias más importantes se dan en que, por un lado, la forma de determinación del aumento del porcentaje de los ingresos brutos no se hace sobre ingresos fijos y variables sino que corresponde a un porcentaje determinado que el concesionario debe pagar al Estado. De otra parte, se define como obligatorio el cumplimiento del plan maestro y por tanto la ejecución de las inversiones a cargo y riesgo del concesionario, definiendo un modelo de ingresos estimados a partir de los cuales se equiparan los ingresos regulados generados.

La cuarta generación de concesiones dio inicio el 30 de diciembre de 2014, con la concesión del Aeropuerto Internacional de Barranquilla-Ernesto Cortissoz. La autoridad pública hizo entrega del aeropuerto al nuevo concesionario en el primer semestre de 2015 (Díaz Olariaga, 2016a).

La política de concesiones de aeropuertos vino acompañada de una política de inversiones. La inversión pública en infraestructura de transporte aéreo ha venido creciendo paulatinamente en la última década hasta llegar a un $14 \%$ como porcentaje del PIB de transporte aéreo en el año 2013, el doble con respecto a una década atrás. Por otro lado, la inversión privada en aeropuertos ha evolucionado de acuerdo a la dinámica propia de las concesiones, pasando de inexistente en el año 1996, año cero de inicio de las primeras concesiones, a un 20\% como porcentaje del PIB de transporte aéreo en el año 2010 (Díaz Olariaga, 2016a). En esta línea, en los últimos años el plan de concesiones generó un importante paquete de inversión privada en casi todos los aeropuertos concesionados, la inversión privada media anual en el periodo 2010-2013 representa el 18\% del PIB del transporte aéreo en el año 2013 (Ministerio de Transporte, 2014).

\section{El mercado de los operadores aéreos}

En lo que se refiere a la liberalización de las tarifas aéreas en Colombia, desde 1992 hasta la expedición de la Resolución 3299 de 2007 de Aerocivil (sobre tarifas nacionales e internacionales de transporte aéreo regular de pasajeros), la intervención de Aerocivil en las tarifas aeronáuticas consistió en la aprobación de las tarifas propuestas por las aerolíneas para una determinada ruta y no en la imposición directa de las mismas. Las tarifas propuestas y sus condiciones debían cumplir con los criterios técnicos de equidad y suficiencia que establecen los Reglamentos Aeronáuticos de Colombia. La Resolución 3299 de 2007 modifica el régimen tarifario mediante eliminación de las tarifas mínimas para las empresas de transporte aéreo nacional e internacional. Dicha resolución establece que las empresas podrán fijar libremente las tarifas, siempre y cuando dichas tarifas no sean superiores a las que Aerocivil haya aprobado a otras aerolíneas en el nivel máximo. El efecto inmediato de la liberalización tarifaria fue la disminución sustancial de las tarifas fijadas por las empresas tanto para los destinos nacionales como internacionales (Díaz Olariaga, 2016b).

En otro orden, en Colombia la entrada al mercado de operadores aéreo privados con un modelo de negocio tradicional, o FSC (Full-Service Carrier), se produjo muy pronto luego de la liberalización del sector (principios de la década de 1990), en 
la actualidad operan cuatro aerolíneas privadas colombianas con un modelo FSC. Pero la entrada al mercado de las líneas aéreas con un modelo de negocio de "bajo costo", o LCC (Low-Cost Carrier), fue muchos años posterior a la liberalización. Las dos únicas LCC que operan, al año 2017, en el mercado colombiano son EasyFly (que inicia operaciones en 2008) y VivaColombia (que inicia operaciones en 2012) (Díaz Olariaga, 2016b). Ambas compañías operan principalmente en el mercado doméstico y con una cuota de mercado (al cierre del año 2015), en conjunto, del 16\% (Aerocivil, 2018).

Y finalmente, mencionar que las tarifas aéreas están totalmente desreguladas desde el año 2012 (Díaz Olariaga y Zea, 2018).

\section{Efectos de la liberalización y de las políticas públicas en el desarrollo del tráfico}

Hay tres hitos que marcan el antes y el después en el desarrollo del transporte aéreo colombiano. El primero, la liberalización del sector del transporte aéreo, iniciada en el año 1991. El segundo, la norma fijada por el gobierno nacional, a través de la Dirección Nacional de Planeación, en el año 1994 (DNP, 1994), la cual establece el reordenamiento institucional y de financiamiento del sector aeronáutico, así como el plan de expansión del sistema aeroportuario, lo que se derivó, entre otras acciones, en el inicio de un programa de concesión privada de varios aeropuertos de la red. Y el tercer hito relevante es la primera definición de un importante paquete de políticas públicas específicas y exclusivas para el sector del transporte aéreo colombiano en el plan nacional de desarrollo 2006-2010 (DNP, 2006).

Entonces, en base a estos hitos, se puede evaluar el principal indicador del sector: el crecimiento de tráfico de pasajeros totales, que entre los años 1992 y 2017 fue de un 863\% (Díaz Olariaga, 2018). La liberalización del sector del transporte aéreo (iniciada en 1991) representó el despegue de la industria, que venía de una década de crecimiento casi nulo. La 1ra y 2da generación de concesiones, en los años 1996 y 2000 respectivamente, no tuvieron un impacto relevante en el crecimiento del transporte aéreo, evento que además se vio perjudicado por la gran crisis económica que experimentó Colombia entre 1999 y 2002. Sin embargo, es a partir del año 2004 cuando empieza un relevante crecimiento del sector del transporte aéreo, cuyo impulso lo da la privatización del principal aeropuerto del país, Bogotá-El Dorado (en el año 2007), posteriormente la 3ra generación de concesiones aeroportuarias (que finalizó en el año 2010), y finalmente la 4ta generación de concesiones, a finales del año 2014, con la concesión del Aeropuerto de Barranquilla (BAQ).

\section{Marco conceptual y datos}

Una serie de tiempo es una secuencia de observaciones de una colección de variables ordenadas a lo largo del tiempo, lo más común es ordenar la serie con unidades de tiempo como días, meses y años, sin embargo es posible que la ordenación sea construida con otras unidades como volumen, latitud, longitud, espacio, entre otros (Morettin y Toloi, 2004). Las técnicas de predicciones cuantitativas son desarrolladas por modelos matemáticos tales como medias móviles, regresión, suavizado exponencial, Box-Jenkins, entre otros. Los modelos de series de tiempo utilizan datos pasados que tienen correlación en el tiempo y son usados como base para realizar pronósticos de resultados en el futuro (Peña, 2010; Melville, 1998). 


\section{Modelos ARIMA de Box-Jenkins}

Los modelos ARIMA (Autoregressive Integrated Moving Average) fueron desarrollados por Box y Jenkins (Box et al., 1994) para series de tiempo estacionarias con auto-dependencia lineal. Se utilizan para analizar las propiedades probabilísticas de las series de tiempo para "permitir que los datos hablen por sí mismos" es decir, se desarrollaron con objetivo de estimar y predecir su comportamiento basado en el pasado de la misma serie. Los pronósticos realizados con los modelos ARIMA tienen la ventaja que no acumulan errores de las variables explicativas como lo hacen los modelos estructurales (Gujarati, 2000). El modelo ARIMA se obtiene por medio de tres componentes: el primero es el componente auto regresiva ("AR") que relaciona la serie con observaciones de periodos anteriores de ella misma (Stock y Watson, 2003). El segundo es el de medias móviles ("MA") que relaciona la serie como una función de una sucesión de errores correspondientes a periodos anteriores ponderados. La tercera parte que corresponde a la "X" en la sigla ARIMAX son las observaciones anteriores de una serie independiente exógena, que en este caso es un indicador de privatización del aeropuerto, el PIB per cápita y la Población.

El modelo integrado auto-regresivo y de medias móviles (ARIMA) es un modelo de fácil ajuste a una serie de tiempo y se basa en datos históricos de series temporales considerando patrones auto-regresivos y de media móvil para producir los pronósticos. Un modelo ARMA (p,q) está representado por la siguiente formula:

$$
\begin{aligned}
& \mathrm{Y}_{\mathrm{t}}=\phi_{1} \mathrm{Y}_{\mathrm{t}-1}+\phi_{2} \mathrm{Y}_{\mathrm{t}-2}+\ldots+\phi_{\mathrm{p}} \mathrm{Y}_{\mathrm{t}-\mathrm{p}}+\varepsilon_{\mathrm{t}}+\theta_{1} \varepsilon_{\mathrm{t}-1}+\theta_{2} \varepsilon_{\mathrm{t}-2}+\ldots+\theta_{\mathrm{q}} \varepsilon_{\mathrm{t}-\mathrm{q}} \\
& \text { donde } \mathrm{Y}_{\mathrm{t}} \text { es la variable dependiente } \\
& \phi \text { son los parámetros auto regresivos } \\
& \theta \text { son los parámetros de medias móviles } \\
& \varepsilon_{\mathrm{t}} \text { se refiere a los errores }
\end{aligned}
$$

Para su utilización se requiere que la serie sea estacionaria, y si no lo es, realizar una diferenciación para transformarla. El modelo diferenciado ARIMA(p,d,q) es el modelo en el cual se realizó una diferenciación y la "d" representa el número de veces que se diferenció la serie. Para representar las ecuaciones se utilizan polinomios de rezago para los parámetros AR y MA de la siguiente forma:

$$
\phi(B)(1-B)^{d} Y_{t}=\theta(B) \varepsilon_{t}
$$

La representación de la metodología Box-Jenkins se muestra en la Figura 1.

Las etapas del diagrama de flujo de la Figura 1 han sido diseñadas para la creación de un modelo ARIMA, sin embargo, las mismas pueden ser aplicadas para la creación de un modelo ARIMAX. Este tipo de modelos además de realizar las estimaciones de los coeficientes contenidos en el modelo ARIMA, adicionalmente se puede estimar los órdenes y los coeficientes de las variables exógenas (Marek, 2000). 


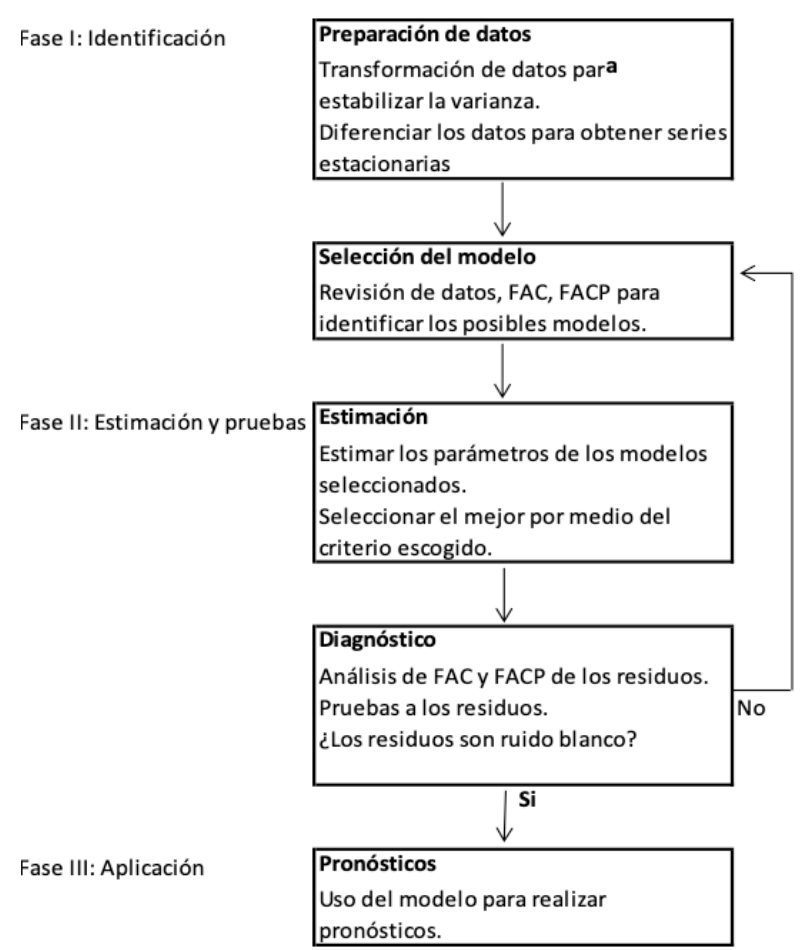

Figura 1. Construcción de un modelo ARIMA/ARIMAX. Fuente: Makridakis et al. (1998).

\section{Modelo ARIMAX}

El modelo ARIMAX (Auto-Regresivo Integrado de Medias Móviles y Entradas Exógenas) es similar al modelo ARIMA descrito anteriormente. La diferencia radica en que el modelo ARIMAX posee además de los parámetros ARIMA por lo menos una variable exógena, variables regresoras independientes que ayudan a explicar el comportamiento de la serie a partir de otras variables que están altamente correlacionadas (Nguyen y Chan, 2005; Pankratz, 1991), de tal manera que para un modelo ARIMAX existirán más de un término a estimar que corresponde al orden de la ecuación que representan las variables exógenas.

Los modelos ARIMAX asumen la siguiente forma (Nguyen y Chan, 2005):

$$
\sum_{j=0}^{\mathrm{p}} \mathrm{di}^{\mathrm{d}} \Delta^{\mathrm{d}} \mathrm{Y}_{\mathrm{t} \mathrm{j}}=\mathrm{b}_{\mathrm{o}}+\sum_{\mathrm{j}=1}^{\mathrm{r}} \mathrm{b}_{\mathrm{j}} \Delta^{\mathrm{d}} \mathrm{X}_{\mathrm{t}, \mathrm{j}}+\sum_{\mathrm{j}=0}^{\mathrm{a}} \mathrm{c}_{\mathrm{j}} \varepsilon_{\mathrm{t}, \mathrm{j}} \text { para } \mathrm{t}=\mathrm{O}, 1,2, \ldots,
$$

donde:

Y es in vector de salidas observables (son puntos de las series de tiempo)

$X$ es un vector de entradas observables

$\varepsilon$ es un vector de elementos que caracteriza ruidos aleatorios no observables

Asumiéndose que $\mathrm{Y}$ y X son estacionarios y $\varepsilon$ tiene media cero y varianza $\sigma_{\varepsilon}^{2}$.

\section{Estimación de los coeficientes del Modelo}

En esta etapa se identifican los coeficientes del modelo seleccionado. Para ello es necesario identificar los coeficientes de la parte auto-regresiva AR y/o de la parte de medias móviles MA, respectivamente, además de la parte correspondiente a las 
variables exógenas. Por lo tanto serán estimados $(\mathrm{p}+\mathrm{q}+1)$ coeficientes ( $p$ para la parte AR; $q$ para la parte MA y 1 para el termino $\varepsilon_{t}$ ). Si la media de la serie $\mu$ no fuera cero, se adiciona una constante al modelo $(\theta)$ teniendo así $(\mathrm{p}+\mathrm{q}+2)$ coeficientes a estimar (Lim y McAleer, 2002).

\section{Pronósticos con el modelo ARIMAX}

Al tener los valores de una serie de tiempo $\mathrm{Y}=\mathrm{Y}_{1} ; \mathrm{Y}_{2} ; \ldots, \mathrm{Y}_{\mathrm{t}}$, en el tiempo $\mathrm{T}=1,2 \ldots$ $\mathrm{t}$, los pronósticos son realizados para valores de $\mathrm{Y}_{\mathrm{t}+\mathrm{h}}$ donde $\mathrm{h} \geq 1$ y el instante $\mathrm{t}$ es llamado el origen del pronóstico (Morettin y Toloi, 2004). El valor pronosticado de $Y_{t+h}$ es dado por . Suponiendo que $W_{t}=Y_{t}$ entonces se utiliza $W_{t}$ en lugar de $Y_{t}$. El valor de $\mathrm{W}_{\mathrm{t}+\mathrm{h}}$ escrito en la forma de la ecuación de diferencias es (Keck et al., 2009; Makridakis et al., 1998):

$$
\mathrm{W}_{\mathrm{t}+\mathrm{h}}=\phi_{1} \mathrm{~W}_{\mathrm{t}+\mathrm{h}-1}+\phi_{2} \mathrm{~W}_{\mathrm{t}+\mathrm{h}-2}+\ldots+\phi_{\mathrm{p}} \mathrm{W}_{\mathrm{t}+\mathrm{h}-\mathrm{p}}+\varepsilon_{\mathrm{t}+\mathrm{h}}-\theta_{1} \varepsilon_{\mathrm{t}+\mathrm{h}-1}-\theta_{2} \varepsilon_{\mathrm{t}+\mathrm{h}-2}-\ldots--\theta_{\mathrm{q}} \varepsilon_{\mathrm{t}+\mathrm{h}-\mathrm{q}}
$$

Para este estudio fueron generados modelos a partir de las series temporales de pasajeros nacionales e internacionales. Se realizaron pruebas de estacionariedad de las series y si no cumplía esta condición se realizaban las diferenciaciones necesarias para convertirlas en estacionarias (Allen y Fildes, 2001). Para encontrar el orden correcto del modelo ARIMAX, se utiliza la metodología propuesta por Box y Jenkins (Box et al., 1994), resumida a continuación:
1. Identificar el orden del modelo ARIMAX (p,d,q,b).
2. Estimar los coeficientes.
3. Validar el modelo.
4. Verificar el ajuste.
5. Seleccionar el mejor modelo disponible.

Las variables que se emplearon en este estudio fueron: series de pasajeros nacionales e internacionales, años de la privatización del aeropuerto, PIB per cápita, población y el impacto (o influencia de la competencia) de las líneas aéreas de bajo costo, a través del número de pasajeros (nacionales) movilizados, siguiendo el análisis de otros autores (Rolim et al., 2016).

\section{Datos}

Como es habitual en los modelos econométricos para pronosticar la demanda de pasajeros aéreos (Singh et al., 2016; Tsui et al., 2014; Scarpel, 2013; Abdelghany y Guzhva, 2010; Suryani et al., 2010; Aleksee y Seixas, 2009), las variables (exógenas) socio-económicas utilizadas en el presente estudio son el PIB per cápita y la población de cada una de las regiones administrativas (que en Colombia se denominan Departamento) en donde se encuentran localizados cada uno de los aeropuertos (privatizados) analizados en el caso de estudio (ver Cuadro 1). Las variables de transporte aéreo consideradas son los pasajeros nacionales e internacionales gestionados por los aeropuertos de estudio. 
Cuadro 1. Datos de aeropuertos privatizados analizados. Fuente: Elaboración propia. Comentario: Los aeropuertos de Barranquilla (BAQ) y Cartagena (CTG) registraron una primera concesión en el año 1996, pero los contratos de concesión implicaron una inversión mínima (simbólica) de las infraestructuras. Mientras que en la segunda (y más reciente) concesión, indicada en la tabla, si se obligó al nuevo operador a una importante inversión para la ampliación y modernización de dichos aeropuertos.

\begin{tabular}{|l|c|c|c|}
\hline Aeropuerto & $\begin{array}{c}\text { Código IATA del } \\
\text { aeropuerto }\end{array}$ & Año de privatización & Departamento \\
\hline Carepa & APO & 2008 & Antioquia \\
\hline Medellín & EOH & 2008 & Antioquia \\
\hline Rionegro & MDE & 2008 & Antioquia \\
\hline Barranquilla & BAQ & $1996 / 2015$ & Atlántico \\
\hline Cartagena & CTG & $1996 / 2010$ & Bolívar \\
\hline Valledupar & VUP & 2010 & Cesar \\
\hline Quibdó & UIB & 2008 & Córdocó \\
\hline Montería & MTR & Bogotá D.C. \\
\hline Bogotá & BOG & 2008 & La Guajira \\
\hline Riohacha & RCH & 2007 & Magdalena \\
\hline Santa Marta & SMR & 2010 & Norte de Santander \\
\hline Cúcuta & CUC & 2010 & $\begin{array}{c}\text { San Andrés y } \\
\text { Providencia }\end{array}$ \\
\hline Providencia & PVA & 2010 & $\begin{array}{c}\text { San Andrés y } \\
\text { Providencia }\end{array}$ \\
\hline San Andrés & ADZ & 2007 & Santander \\
\hline Barrancabermeja & EJA & 2007 & Santander \\
\hline Bucaramanga & BGA & 2010 & Sucre \\
\hline Corozal & CZU del Cauca \\
\hline Cali & CLO & 2010 & \\
\hline
\end{tabular}

La base de datos con información histórica del número de pasajeros en los 18 aeropuertos en estudio es de suma importancia para el ejercicio de pronósticos. Los datos recolectados son empleados como punto de partida para la selección del mejor modelo que pronostique el número de pasajeros a corto plazo. Los datos de series de tiempo utilizados en este trabajo fueron obtenidos de las siguientes fuentes (todas instituciones públicas de Colombia): el PIB per cápita se obtuvo del Banco de la República (2018), los datos de población del Departamento Administrativo Nacional de EstadísticaDANE (2018), y los datos aeronáuticos, o de transporte aéreo (pasajeros nacionales e internacionales), de la Aeronáutica Civil de Colombia (Aerocivil, 2018), la variable año de privatización es una variable Dummy que toma valores 1 a partir del año de privatización. El histórico de datos consolidados cubre un periodo de 24 años (19922015), que inicia al año siguiente de la liberalización del transporte aéreo en Colombia (Díaz Olariaga, 2016a).

\section{Resultados y análisis}

Las series de tiempo resultantes se muestran en el Cuadro 2, donde se aprecian los modelos resultantes para los 18 aeropuertos, junto a la estimación de parámetros de las variables exógenas empleadas. En dicho cuadro se puede observar que en algunos aeropuertos la variable población no fue significativa al modelo por lo tanto la variable no tiene parámetro estimado; lo mismo se verifica para el impacto de la competencia de las líneas de aéreas de bajo costo (no significativa), debido a que 
no se dispone de suficientes datos históricos, como se dijo anteriormente dichas (dos) compañías empezaron a operar recientemente, 2008 y 2012 respectivamente (Díaz Olariaga, 2016b), y en el presente su peso en el mercado es aún muy bajo (Aerocivil, 2018).

Las variables intervención (año de privatización del aeropuerto), PIB per cápita y población aportan información al modelo de pronósticos de pasajeros puesto que estas variables se encuentran asociadas al comportamiento de la serie pasajeros. Los resultados muestran que el parámetro asociado a la variable privatización del aeropuerto es mayor a 1 en casi todos los aeropuertos, lo que se traduce en aumento de pasajeros a partir del año de privatización, lo mismo ocurre con el PIB per cápita y la población, dado que cuando estas variables aumentan su valor lo mismo ocurre con el número de pasajeros.

En el Cuadro 2 se encuentran los modelos de series de tiempo que se emplearon para ajustar los datos de pasajeros a nivel nacional así como las estimaciones de los parámetros de las variables exógenas.

Cuadro 2. Modelos de series de tiempo y parámetros estimados de las variables auxiliares, pasajeros nacionales. Fuente: Elaboración propia.

\begin{tabular}{|c|c|c|c|c|}
\hline \multirow[t]{2}{*}{ Aeropuerto } & \multirow[t]{2}{*}{ Modelo } & \multicolumn{3}{|c|}{ Estimación de parámetros } \\
\hline & & $\begin{array}{c}\text { Intervención } \\
\text { (privatización } \\
\text { del } \\
\text { aeropuerto) }\end{array}$ & $\begin{array}{l}\text { PIB per } \\
\text { Cápita }\end{array}$ & Población \\
\hline CAREPA & Arimax $(6,1,1)+$ inte + pbx & 1,10 & 1,14 & \\
\hline MEDELLIN & $\operatorname{Arimax}(4,1,4)+$ inte + pbx & 1,21 & 1,27 & \\
\hline RIONEGRO & $\operatorname{Arimax}(1,1,1)+$ inte + pbx & 1,14 & 1,24 & \\
\hline BARRANQUILLA & Arimax $(3,1,1)+$ inte + pbx+Pob & 1,04 & 1,10 & 26,06 \\
\hline CARTAGENA & $\operatorname{Arimax}(3,1,1)+$ inte $+\mathrm{pbx}$ & 1,24 & 1,32 & \\
\hline VALLEDUPAR & Arimax $(3,1,1)+$ inte $+p b x+$ Pob & 1,21 & 1,01 & 19,41 \\
\hline QUIBDO & Arimax $(0,1,1)+$ inte $+p b x+P o b$ & 1,14 & 1,38 & 6,78 \\
\hline MONTERIA & Arimax $(0,1,1)+$ inte $+p b x+P o b$ & 1,27 & 1,35 & 17,93 \\
\hline BOGOTA & Arimax $(0,1,1)+$ inte + pbx & 1,02 & 1,24 & \\
\hline RIOHACHA & $\operatorname{Arimax}(0,1,1)+$ inte $+p b x$ & 1,02 & 1,47 & \\
\hline SANTA MARTA & $\operatorname{Arimax}(3,1,1)+$ inte $+p b x+P o b$ & 1,40 & 1,36 & 24,83 \\
\hline CUCUTA & $\operatorname{Arimax}(5,1,1)+$ inte + pbx & 1,37 & 1,63 & \\
\hline PROVIDENCIA & Arimax $(1,1,1)+$ inte $+p b x$ & 1,01 & 1,05 & \\
\hline SAN ANDRES & $\operatorname{Arimax}(1,1,1)+$ inte+pbx & 1,08 & 1,20 & \\
\hline BARRANCABERMEJA & $\operatorname{Arimax}(4,1,1)+$ inte + pbx & 0,93 & 1,51 & \\
\hline BUCARAMANGA & $\operatorname{Arimax}(0,1,1)+$ inte+pbx & 1,32 & 1,09 & \\
\hline COROZAL & $\operatorname{Arimax}(5,1,1)+$ inte $+p b x$ & 0,91 & 1,52 & \\
\hline CALI & Arimax $(3,1,1)+$ inte $+p b x$ & 0,92 & 1,53 & \\
\hline
\end{tabular}

El parámetro estimado "Intervención del Aeropuerto" se interpreta como el incremento o decrecimiento en los pasajeros a partir del año de la privatización del mismo.

En el Cuadro 3 (a, b, c) se encuentra la estimación de las tasas de crecimiento para pasajeros nacionales en los 18 aeropuertos, usando el modelo ARIMAX. 
Cuadro 3 (a, b, c). Estimación de crecimiento de pasajeros nacionales para los aeropuertos en estudio.

Fuente: elaboración propia.

\begin{tabular}{|l|l|l|l|l|l|l|}
\hline \multicolumn{7}{|l|}{ Aeropuertos más importantes de Colombia por el número de pasajeros transportados } \\
\hline AEROPUERTO & $\mathbf{2 0 1 6}$ & $\mathbf{2 0 1 7}$ & $\mathbf{2 0 1 8}$ & $\mathbf{2 0 1 9}$ & $\mathbf{2 0 2 0}$ & $\mathbf{2 0 2 1}$ \\
\hline BOGOTA & $2,60 \%$ & $0,90 \%$ & $3,20 \%$ & $3,10 \%$ & $3,90 \%$ & $2,00 \%$ \\
\hline RIONEGRO & $4,30 \%$ & $6,80 \%$ & $5,10 \%$ & $2,30 \%$ & $1,80 \%$ & $4,40 \%$ \\
\hline MEDELLIN & $-0,90 \%$ & $1,10 \%$ & $-1,50 \%$ & $2,30 \%$ & $1,50 \%$ & $2,50 \%$ \\
\hline CALI & $6,90 \%$ & $6,10 \%$ & $5,40 \%$ & $4,60 \%$ & $7,70 \%$ & $3,50 \%$ \\
\hline SANTA MARTA & $11,10 \%$ & $3,30 \%$ & $10,20 \%$ & $7,50 \%$ & $5,20 \%$ & $5,50 \%$ \\
\hline
\end{tabular}

\begin{tabular}{|l|l|l|l|l|l|l|}
\hline \multicolumn{7}{|l|}{ Aeropuertos en segundo lugar de importancia por número de pasajero transportados } \\
\hline CARTAGENA & $6,40 \%$ & $2,10 \%$ & $4,60 \%$ & $2,90 \%$ & $1,50 \%$ & $2,20 \%$ \\
\hline BARRANQUILLA & $-5,40 \%$ & $6,60 \%$ & $7,80 \%$ & $7,00 \%$ & $5,30 \%$ & $7,60 \%$ \\
\hline SAN ANDRES & $2,20 \%$ & $8,80 \%$ & $1,10 \%$ & $2,80 \%$ & $0,80 \%$ & $1,30 \%$ \\
\hline BUCARAMANGA & $4,10 \%$ & $4,80 \%$ & $5,80 \%$ & $5,90 \%$ & $5,50 \%$ & $6,20 \%$ \\
\hline CUCUTA & $52,80 \%$ & $0,50 \%$ & $2,90 \%$ & $2,00 \%$ & $2,10 \%$ & $1,90 \%$ \\
\hline
\end{tabular}

\begin{tabular}{|l|l|l|l|l|l|l|}
\hline \multicolumn{7}{|l|}{ Aeropuertos en tercer lugar de importancia por número de pasajeros transportados } \\
\hline MONTERIA & $9,20 \%$ & $-2,10 \%$ & $2,10 \%$ & $4,00 \%$ & $4,00 \%$ & $5,40 \%$ \\
\hline QUIBDO & $10,60 \%$ & $3,20 \%$ & $4,80 \%$ & $6,10 \%$ & $3,30 \%$ & $2,10 \%$ \\
\hline VALLEDUPAR & $2,40 \%$ & $2,60 \%$ & $2,40 \%$ & $2,30 \%$ & $2,20 \%$ & $2,00 \%$ \\
\hline CAREPA & $11,20 \%$ & $-2,90 \%$ & $1,70 \%$ & $-4,70 \%$ & $-0,40 \%$ & $0,70 \%$ \\
\hline BARRANCABERMEJA & $-13,40 \%$ & $-15,40 \%$ & $-17,90 \%$ & $-13,50 \%$ & $-9,80 \%$ & $-4,60 \%$ \\
\hline RIOHACHA & $-15,00 \%$ & $-24,60 \%$ & $-19,60 \%$ & $-11,50 \%$ & $3,70 \%$ & $14,50 \%$ \\
\hline COROZAL & $18,90 \%$ & $15,90 \%$ & $-9,60 \%$ & $-9,10 \%$ & $-5,00 \%$ & $-3,90 \%$ \\
\hline PROVIDENCIA & $0,90 \%$ & $0,30 \%$ & $0,20 \%$ & $0,20 \%$ & $0,20 \%$ & $0,20 \%$ \\
\hline
\end{tabular}

En las Figuras 2, 3, y 4 se representa gráficamente el pronóstico del crecimiento porcentual de los aeropuertos en estudio.

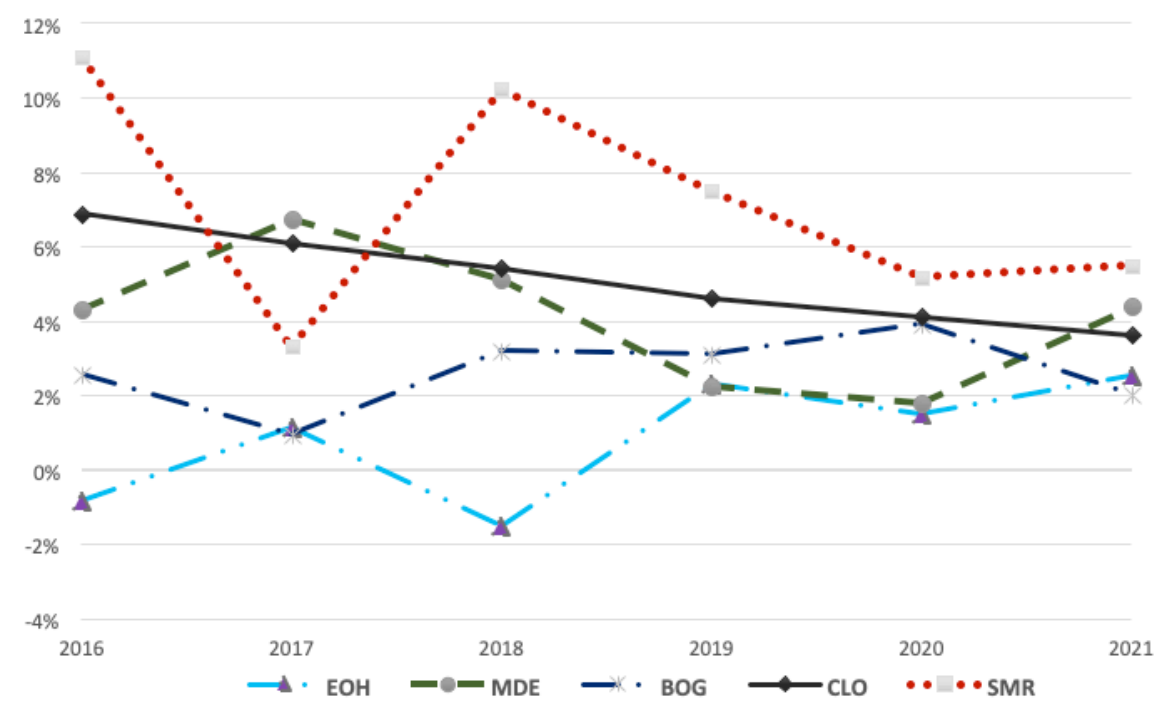

Figura 2. Pronóstico de crecimiento (\%) de pasajeros nacionales, 1er. grupo de aeropuertos en estudio. Fuente: Elaboración propia. 
En la Figura 2 se observa un crecimiento positivo, en todos los años de pronóstico, para todos los aeropuertos del grupo analizado (los más importantes de Colombia por número de pasajeros transportados), excepto el aeropuerto de Medellín (EOH), aunque sólo en un par de años. Es probable, y según ciertos estudios sobre competencia entre aeropuertos (IATA, 2013; Thelle et al., 2012; Fröhlich y Niemeier, 2001; Forsyth et al., 2010), que esta situación se deba a los aeropuertos EOH (Medellín) y MDE (Rionegro), en cierta medida, "compiten entre sí", ya que se encuentran en ciudades muy próximas (limítrofes) en el mismo departamento administrativo.

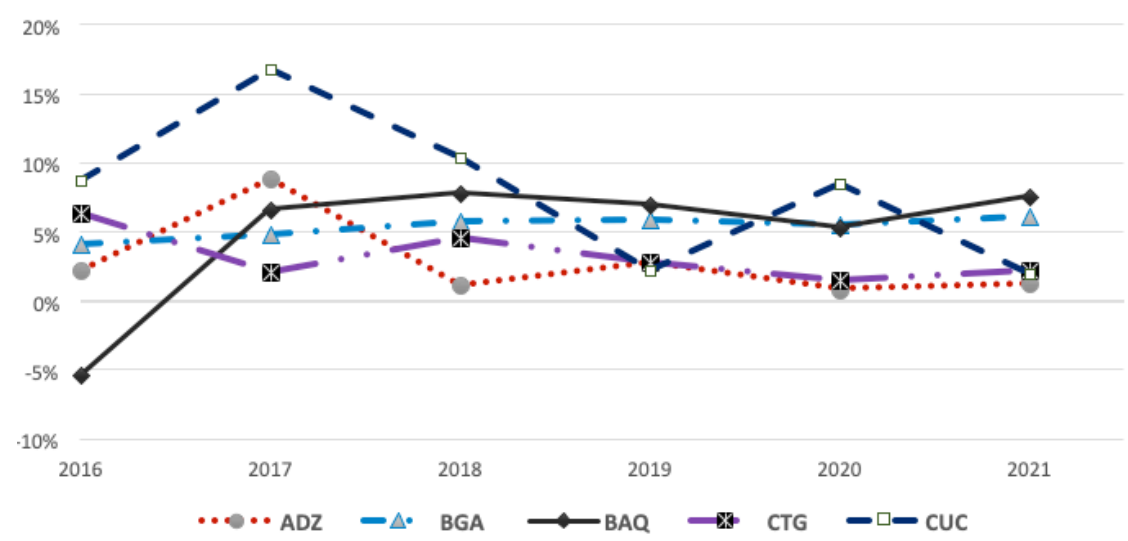

Figura 3. Pronóstico de crecimiento (\%) de pasajeros nacionales, 2do. grupo de aeropuertos en estudio. Fuente: Elaboración propia.

En el grupo de aeropuertos de la Figura 3 (segundo en importancia en cuanto a volumen de pasajeros transportados) se aprecia un crecimiento positivo, en el periodo de pronóstico, en todos los aeropuertos excepto en el de Barranquilla (BAQ), y sólo para el año 2016. Esto es coherente con la situación de dicho aeropuerto, el cual, como se dijo en una sección anterior, fue concesionado finalizando 2014 y estará en proceso de ampliación y remodelación durante el periodo 2015-2018, lo que puede afectar a su operatividad normal.

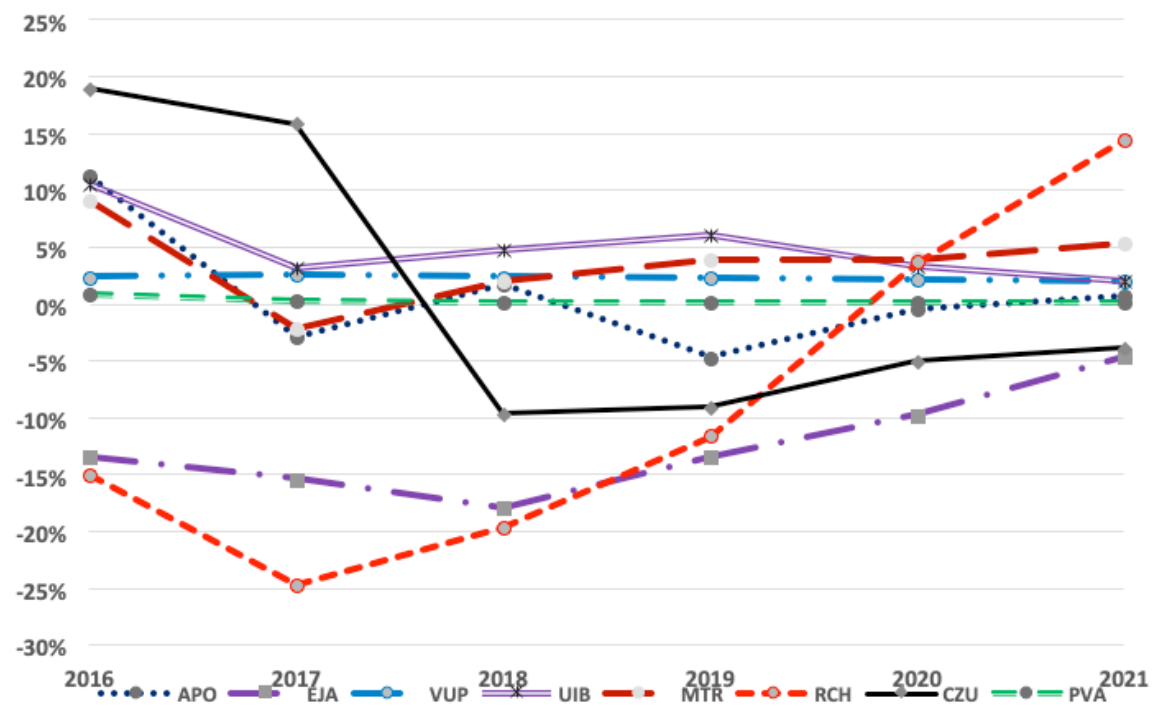

Figura 4. Pronóstico de crecimiento (\%) de pasajeros nacionales, zer. grupo de aeropuertos en estudio. Fuente: Elaboración propia. 
En la Figura 4 se analiza el último grupo de aeropuertos privatizados que tienen en común su tamaño, son todos de tipo pequeño, en lo que se refiere al tráfico (doméstico) de pasajeros que gestionan anualmente (son el grupo de aeropuertos más pequeños, por número de pasajeros transportados, de todos los concesionados). Como se aprecia en la Figura 4, la mayoría de los aeropuertos presenta un pronóstico de crecimiento positivo muy modesto, o incluso nulo, en el periodo de estudio, a excepción de los siguientes aeropuertos: Barrancabermeja (EJA), en el corazón de la zona petrolera colombiana, industria que se ha visto fuertemente afectada por la caída del precio del petróleo en los últimos años (Toro et al., 2015); Corozal (CZU), que aunque se encuentra en la zona turística colombiana compite fuertemente con otros aeropuertos muy próximos (Montería (MTR) y Cartagena de Indias (CTG)); Riohacha ( $\mathrm{RCH})$, situada en uno de los departamentos administrativos más pobres de Colombia, y con un índice de crecimiento económico muy por debajo de la media del país (Banco de República, 2018); y finalmente Carepa (APO), un aeropuerto regional pequeño (que sirve a una ciudad muy pequeña), y en donde la economía de su zona de influencia es primordialmente agrícola-ganadera.

En el Cuadro 4 se encuentran los modelos de series de tiempo que se emplearon para ajustar los datos de pasajeros internacionales así como las estimaciones de los parámetros de las variables exógenas. Sólo seis de los aeropuertos en estudio tienen carácter internacional. Y en el Cuadro 5 se presentan las estimaciones de crecimiento porcentual de pasajeros internacionales de los aeropuertos (internacionales) en estudio.

Cuadro 4. Modelos de series de tiempo por aeropuerto y parámetros estimados de las variables auxiliares para pasajeros internacionales. Fuente: Elaboración propia.

\begin{tabular}{|l|l|c|c|}
\hline \multirow{2}{*}{ Aeropuerto } & \multirow{2}{*}{ Modelo } & \multicolumn{2}{|c|}{ Estimación de parámetros } \\
\cline { 3 - 4 } & & $\begin{array}{l}\text { Intervención (privatización } \\
\text { del aeropuerto) }\end{array}$ & PIB per cápita \\
\hline RIONEGRO & Arimax(1,1,1)+inte+pbx & 1,09 & 1,12 \\
\hline BARRANQUILLA & Arimax(o,1,7)+inte+pbx & 1,18 & 1,25 \\
\hline CARTAGENA & Arimax(o,1,1)+inte+pbx & 1,08 & 1,59 \\
\hline BOGOTA & Arimax $(1,1,1)+$ inte+pbx & 1,03 & 1,27 \\
\hline BUCARAMANGA & Arimax $(4,1,1)+$ inte+pbx & 1,11 & 4,43 \\
\hline CALI & Arimax $(4,1,1)+$ inte+pbx & 1,03 & 1,69 \\
\hline
\end{tabular}

Cuadro 5. Estimación de crecimiento de pasajeros internacionales, aeropuertos en estudio. Fuente: Elaboración propia.

\begin{tabular}{|l|c|c|c|c|c|c|}
\hline AEROPUERTO & $\mathbf{2 0 1 6}$ & $\mathbf{2 0 1 7}$ & $\mathbf{2 0 1 8}$ & $\mathbf{2 0 1 9}$ & $\mathbf{2 0 2 0}$ & $\mathbf{2 0 2 1}$ \\
\hline RIONEGRO & $8,87 \%$ & $8,90 \%$ & $8,85 \%$ & $8,81 \%$ & $8,77 \%$ & $8,76 \%$ \\
\hline BARRANQUILLA & $1,47 \%$ & $-0,05 \%$ & $1,51 \%$ & $1,23 \%$ & $0,92 \%$ & $0,73 \%$ \\
\hline CARTAGENA & $5,11 \%$ & $2,46 \%$ & $2,18 \%$ & $2,19 \%$ & $2,14 \%$ & $1,93 \%$ \\
\hline BOGOTA & $6,30 \%$ & $4,96 \%$ & $3,95 \%$ & $3,23 \%$ & $2,69 \%$ & $2,22 \%$ \\
\hline BUCARAMANGA & $6,44 \%$ & $10,83 \%$ & $6,83 \%$ & $7,09 \%$ & $8,64 \%$ & $7,53 \%$ \\
\hline CALI & $2,45 \%$ & $2,96 \%$ & $2,17 \%$ & $2,66 \%$ & $1,97 \%$ & $1,90 \%$ \\
\hline
\end{tabular}

En la Figura 5 se presenta gráficamente las estimaciones de crecimiento porcentual de los pasajeros internacionales de los aeropuertos en estudio. Como se puede apreciar, todos los aeropuertos verifican un pronóstico positivo de crecimiento en el periodo analizado, con excepción del aeropuerto de Barranquilla (BAQ), que como se mencionó con anterioridad, se encuentra en proceso de remodelación / ampliación /modernización en el periodo 2015-2018; asimismo no presenta crecimiento negativo, sólo un crecimiento nulo en el año 2017. 


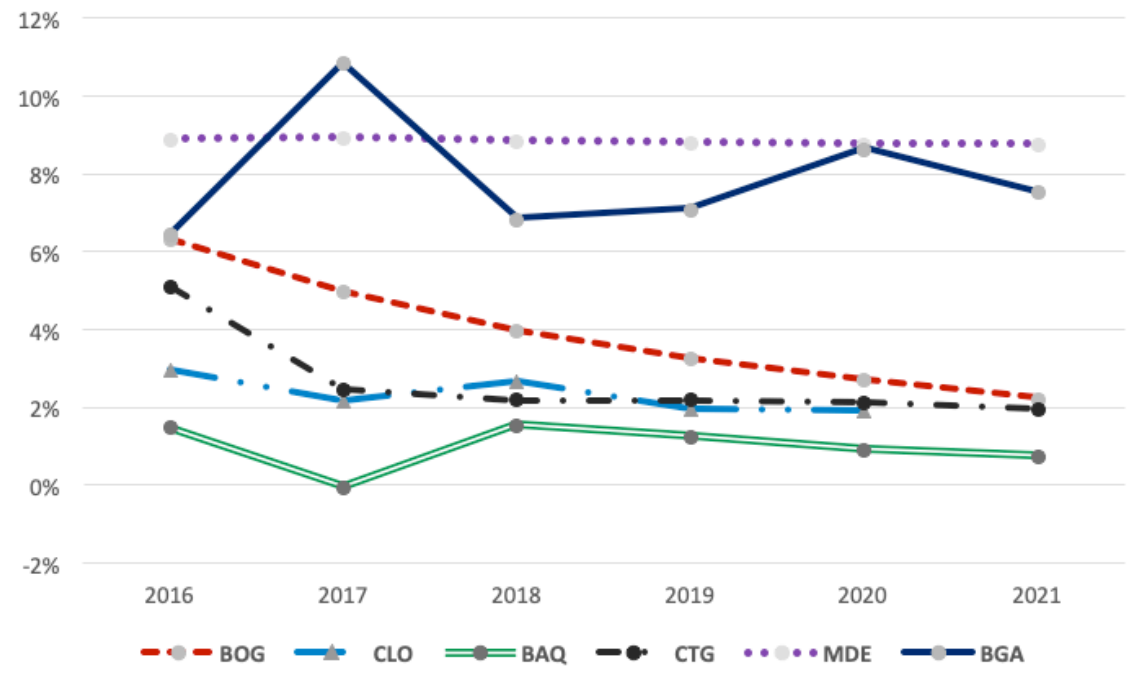

Figura 5. Pronóstico de crecimiento (\%) de pasajeros internacionales de aeropuertos en estudio. Fuente: Elaboración propia.

\section{Conclusiones}

El presente trabajo utilizó un modelo econométrico para pronosticar la demanda de pasajeros nacionales e internacionales, a corto plazo, en aeropuertos privatizados, y se utilizó como caso de aplicación la situación aeroportuaria de Colombia. El objetivo era estimar el impacto de la privatización del aeropuerto en el pronóstico de la demanda de transporte aéreo; el modelo desarrollado utilizó para ello variables aeronáuticas (pasajeros), socio-económicas (PIB/cápita y población) y de competencia (influencia de las líneas aéreas de bajo costo), además del factor de "intervención" del aeropuerto (año de su privatización).

Los principales resultados de la investigación fueron los siguientes: a) en el $62 \%$ de los aeropuertos analizados (donde están incluidos los más importantes, por volumen de pasajero transportado de toda la red) se pronostica un crecimiento positivo de tráfico nacional en todos los años del periodo analizado, para el 38\% de aeropuertos restante se pronostica un crecimiento negativo sólo en algunos años en el periodo analizado. Este segundo grupo de aeropuertos (de crecimiento negativo parciales) tienen en común que son los más pequeños de los (aeropuertos) privatizados, sirven a ciudades relativamente pequeñas y están en regiones cuyo nivel económico (o de riqueza) está por debajo de la media nacional, a excepción de situaciones puntuales, ya argumentadas en el texto, como son los casos de los aeropuertos de Medellín (EOH) y Barranquilla (BAQ); b) para el caso del tráfico de pasajeros internacionales, se pronostica un crecimiento positivo para todos los aeropuertos (internacionales) analizados y para todos los años del periodo analizado; el único aeropuerto que verifica un pronóstico de crecimiento casi nulo, y sólo para un año, es el aeropuerto de Barranquilla (BAQ), cuya situación se fundamenta en el texto; c) estos resultados van en la línea de otros estudios, tanto a nivel global como regional, donde se afirma que la privatización produce un aumento general de la demanda de los aeropuertos privatizados, un fenómeno vinculado a una gestión privada más flexible y con una mejor estrategia en el desarrollo de nuevas rutas, tanto con las compañías aéreas existentes, como con nuevas aerolíneas; y finalmente c) en lo que refiere a las líneas aéreas de bajo costo, no se pudo medir su influencia en el pronóstico de la demanda, debido, principalmente, al poco tiempo de operación 
en Colombia (de las dos actualmente existente, la más importante empezó a operar a mediados de 2012), y esta investigación utiliza datos consolidados a cierre del año 2015; además, y como se mencionó en el texto, la participación de dichas aerolíneas en el mercado (sólo doméstico) es muy reducida.

En definitiva, la evidencia encontrada en este trabajo señala que la privatización, en principio, parece ser una solución exitosa en la gestión de aeropuertos de un país en vías de desarrollo, como es el caso de Colombia, al menos desde el punto de vista de la demanda. Esta afirmación se soporta con la evidencia de que como resultado de las políticas públicas, tanto de privatización como de inversión (pública y privada) en infraestructura aeroportuaria, en los últimos 25 años el transporte de pasajeros (totales) creció aproximadamente un 863\% (Díaz Olariaga, 2018). 


\section{Q Bibliografía}

» Abed S.Y.; Ba-Fail, A.O. y Jasimuddin S.M. (2001). An econometric analysis of international air travel demand in Saudi Arabia. Journal of Air Transport Management, 7, 143-148.

" Advani, A. y Borins, S. (2001). Managing airports: a test of the new public management. International Public Management Journal, 4, 91-107.

»Aerocivil(2018). Estadísticas. http://www.aerocivil.gov.co/atencion/estadisticasde-las-actividades-aeronauticas, Consulta: 22 enero 2018

» Allen, G. y Fildes, R. (2001). Econometric forecasting. In Armstrong, J.S. (ed.), Principles of Forecasting. Norwell, MA: Kluwer Academic Publishers.

» Banco de la República de Colombia (2018). Estadísticas. http://www.banrep. gov.co/es/-estadisticas

» Bettini, H.F.A.J. y Oliveira, A.V.M. (2016). Two-sided platforms in airport privatization. Transportation Research Part E, 93, 262-278.

"Bosch, A. y García Montalvo, J. (2003). Free and Nondiscriminatory Access to Airports: A Proposal for Latin America. Working paper, Inter-American Development Bank, Washington D.C.

» Box, G.E.P.; Jenkins G.M. y Reinsel, G.C. (1994). Time Series Analysis: Forecasting and Control. San Francisco: Holden-day.

»CAF (2010). Infraestructura públicay participación privada: conceptosyexperiencias en América y España. Caracas: Ed. Corporación Andina de Fomento-CAF.

»Carvallo, C. (2008). Experiencia chilena en concesiones aeroportuarias. Santiago de Chile: Ministerio de Obras Públicas.

»DANE (2018). Estadísticas. https://www.dane.gov.co/index.php/estadisticaspor-tema Consulta: 2 Febrero 2018.

"Díaz Olariaga, O. y Zea, J.F. (2018). Influence of the liberalization of the air transport industry on configuration of the traffic in the airport network. Transportation Research Procedia, 33, 43-50.

" Díaz Olariaga, O. (2018). Prognosis de tráfico aéreo en contexto de postliberalización del transporte aéreo. Caso de Colombia. Working Paper. DOI: 10.13140/RG.2.2.11443.73760

"Díaz Olariaga, O. (2016a). Análisis de la evolución de las políticas públicas y de regulación en la industria aeroportuaria en Colombia. Documentos y Aportes en Administración Pública y Gestión Estatal, 26, 7-42.

"Díaz Olariaga, O. (2016b). Análisis del desarrollo reciente del transporte aéreo en Colombia. Revista Transporte y Territorio, 14, 122-143.

" DNP (1994). Reordenamiento institucional y plan de expansión del sistema aeroportuario - Documento CONPES 2727. Bogotá: Departamento Nacional de Planeación.

"Espirito Santo, R.A. (2013). Airport privatization and Business Models: What Brazil has and what Brazil Needs. VI WALA Conference, Montreal, Canada. September 04-06, 2013. 
» Forsyth, P., Gillen, D., Müller, J. y Niemeier. H. (2010). Airport competition. Burlington: Ashgate Publishing.

"Freestone, R. y Baker, D. (2010). Challenges in land use planning around Australian airports. Journal of Air Transport Management, 16, 264-271.

» Fröhlich, K. y Niemeier, H. (2011). The importance of spatial economics for assessing airport competition. Journal of Air Transport Management, 17, 44-48.

" Gong, S.X.H., Cullinane, K. y Firth, M. (2012). The impact of airport and seaport privatization on efficiency and performance: Are view of the international evidence and implications for developing countries. Transport Policy, 24, 37-47.

»Gujarati, D.N. (2000). Econometria Básica. São Paulo: Makron Books.

" IATA (2013). Airport competition. Geneva: IATA.

"ICAO (2003). Background of Liberalization and experiences in the Latin American Region. Worldwide Air Transport Conference ATConf/5-WP/98, Montreal, 24 to 29 March 2003.

" Keck, A., Raubold, A. y Truppia, A. (2009). Forecasting International Trade: A Time Series Approach. OECD Journal: Journal of Business Cycle Measurement and Analysis, 9(2), 157-176.

»Lim, C. y McAleer, M. (2002). Time series forecasts of international travel demand for Australia. Tourism Management, 23, 389-396.

» Lin, M.H. (2013). Airport privatization in congested hub-spoke networks. Transportation Research Part B, 54, 51-67.

» Lipovich, G. (2008). The privatization of Argentine airports. Journal of Air Transport Management, 14, 8-15.

» Makridakis, S., Wheelwright, S.C. y Hyndman, R.J. (1998). Forecasting: Methods and Applications. New York: John Wiley \& Sons.

»Marek, L. (2000). Transfer function models. Acta Oeconomica Pragnesia, 8(3), 83-94.

" Melville, J.A. (1998). An empirical model of the demand for international air travel for the Caribbean region. International Journal of Transport Economics, $\mathrm{XXV(3),} \mathrm{313-336.}$

»Mendiola, A., Arévalo, G., Maratuech, P., Pérez, J. y Valencia, J.C. (2011). Concesión del aeropuerto Jorge Chávez: evaluación del valor generado. Lima: ESAN Ediciones.

" Ministerio de Transporte (2014). Transporte en cifras, estadísticas 2013. Bogotá: Ministerio de Transporte de Colombia.

» Morettin, P.A. y Toloi, C.M.C. (2004). Análise de Séries Temporais. São Paulo: Blucher.

» Nguyen, H.H. y Chan, C.W. (2005). Applications of data analysis techniques for oil production prediction. Engineering Applications of Artificial Intelligence, 18, 549-558.

"Noruzoliaee, M., Zou, B. y Zhang, A. (2015). Airport partial and full privatization in a multi-airport region: focus on pricing and capacity. Transportation Research Part E, 77, 45-60.

» Oum, T.H., Yan, J. y Yu, C. (2008). Ownership forms matter for airport efficiency: a stochastic frontier investigation of worldwide airports. Journal of Urban Economics, 64(2), 422-435. 
» Oum, T.H., Adler, N. y Yu, C. (2006). Privatization, corporatization, ownership forms and their effects on the performance of the world's major airports. Journal of Air Transport Management, 12(3), 109-121.

»Pankratz, A. (1991). Forecasting with Dynamic Regression Models. New York: Wiley \& Sons.

»Parker, D. (1999).The performance of BAA before and after privatization. Journal of Transport Economics and Policy, 33, 133-145.

"Peña, D. (2010). Análisis de series temporales. Madrid: Alianza Editorial.

» Rico Galeana, O. (2008). The privatization of Mexican airports. Journal of Air Transport Management, 14, 320-323.

" Rolim, P.S.W., Bettini, H.F.A.J. y Oliveira, A.V.M. (2016). Estimating the impact of airport privatization on airline demand: A regression-based event study. Journal of Air Transport Management, 54, 31-41.

» Scarpel, R.A. (2013). Forecasting air passengers at São Paulo International Airport using a mixture of local experts model. Journal of Air Transport Management, 26, 35-39.

"Scotti, D., Malighetti, P., Martini, G. y Volta, N. (2012). The impact of airport competition on technical efficiency: a stochastic frontier analysis applied to Italian airport. Journal of Air Transport Management, 22, 9-15.

"Serebrisky, T. (2012). Airport Economics in Latin America and the Caribbean. Washington D.C.: The World Bank.

» SIC - Superintendencia de Industria y Comercio de Colombia - (2013). Estudios de Mercado, Aeropuertos de Colombia (2010-2012). Bogotá: SIC.

"Singh, D.P., Dalei, N.N. y Raju, B. (2016). Forecasting investment and capacity addition in Indian airport infrastructure: Analysis from post-privatization and post-economic regulation era. Journal of Air Transport Management, 53, 218-225.

» Stock, J.H. y Watson, M.W. (2003). Introduction to Econometrics. New York: Addison Wesley.

» Sun, Y. y Schönfeld, P. (2015). Stochastic capacity expansion models for airport facilities. Transportation Research Part B, 80, 1-18.

» Thelle, M.H., Pedersen, T.T. y Harhoff, F. (2012). Airport Competition in Europe. Copenhagen: Copenhagen Economics.

" Toro, J., Garavito, A., López, D.C. y Montes, E. (2015). El choque petrolero y sus implicaciones en la economía colombiana. Bogotá: Banco de la República.

"Zhang, A. y Zhang, Y. (2003). Airport charges and capacity expansion: effects of concessions and privatization. Journal of Urban Economics, 53(1), 54-75.

\section{Oscar Díaz Olariaga / oscardiazolariaga@usantotomas.edu.co}

Ingeniero Aeronáutico por la Universidad Nacional de Córdoba (Argentina); Doctor Ingeniero Aeronáutico por la Universidad Politécnica de Madrid (España); y Doctor en Ciencias Económicas y Empresariales por la UNED (España). Docente e investigador en la Facultad de Ingeniería Civil, Universidad Santo Tomás (Bogotá, Colombia). Campo de investigación: transporte aéreo. 


\section{Edwin Girón Amaya / edwingiron@usantotomas.edu.co}

Estadístico de la Universidad Nacional de Colombia; Mestre em Estatística de la Universidad Federal de Pernambuco (Brasil). Docente e investigador en la Facultad de Estadística, Universidad Santo Tomás (Bogotá, Colombia). Campo de investigación: estadística no paramétrica, muestreo, procesamiento de imágenes satelitales. 